\title{
LiNbTeO $_{5}$ : A High-performance Multifunctional Crystal Material with Very Large Second Harmonic Generation Response and Piezoelectric Coefficient
}

Kaichuang Chen, ${ }^{\dagger}, \xi$ Chensheng Lin, ${ }^{\S}$ Guang Peng, ${ }^{\dagger}, \xi, *$ Yu Chen ${ }^{\dagger}$ Haizhou Huang, ${ }^{\dagger}$ Enze Chen, ${ }^{\dagger}$ Yunxiao Min, ${ }^{\dagger}$ Tao Yan, ${ }^{\dagger}$ Min Luo ${ }^{\dagger}$ and Ning $\mathrm{Ye}^{\star * *}$

${ }^{\dagger}$ Key Laboratory of Optoelectronic Materials Chemistry and Physics, Fujian Institute of Research on the Structure of Matter, Chinese Academy of Sciences, Fuzhou, Fujian 350002, China

$\$$ Tianjin Key Laboratory of Functional Crystal Materials, Institute of Functional Crystal, Tianjin University of Technology, Tianjin 300384, China

$\S$ State Key Laboratory of Structural Chemistry, Fujian Institute of Research on the Structure of Matter, Chinese Academy of Sciences, Fuzhou, Fujian 350002, China

$\xi$ University of Chinese Academy of Sciences, Beijing 100049, China

E-mail: gpeng@fjirsm.ac.cn; nye@fjirsm.ac.cn 
Table S1. Crystal data and structural refinement for $\mathrm{LiNbTeO}_{5}$.

\begin{tabular}{|c|c|}
\hline Formula & $\mathrm{LiNbTeO}_{5}$ \\
\hline Formula weight & 307.45 \\
\hline Temperature (K) & 293.15 \\
\hline$\lambda(\AA)$ & 0.71073 \\
\hline Crystal system & Monoclinic \\
\hline Space group & $\mathrm{P} 2_{1}$ \\
\hline $\mathrm{a}(\AA)$ & $5.122(6)$ \\
\hline $\mathrm{b}(\AA)$ & $10.243(11)$ \\
\hline $\mathrm{c}(\AA)$ & 7.814(9) \\
\hline$\alpha(\mathrm{deg})$ & 90 \\
\hline$\beta(\operatorname{deg})$ & 91.923(19) \\
\hline$\gamma(\mathrm{deg})$ & 90 \\
\hline $\mathrm{V}\left(\AA^{3}\right)$ & $409.7(8)$ \\
\hline Z & 4 \\
\hline$\rho($ calcd $)\left(\mathrm{g} / \mathrm{cm}^{3}\right)$ & 4.984 \\
\hline$\mu\left(\mathrm{mm}^{-1}\right)$ & 9.816 \\
\hline $\mathrm{F}(000)$ & 544 \\
\hline \multirow[t]{2}{*}{$\theta(\mathrm{deg})$} & $2.608-26.042$ \\
\hline & $-6 \leq \mathrm{h} \leq 6$ \\
\hline \multirow[t]{2}{*}{ Index range } & $-12 \leq \mathrm{k} \leq 12$ \\
\hline & $-9 \leq 1 \leq 9$ \\
\hline Reflections collected / unique & $2679 / 1468$ \\
\hline $\mathrm{R}_{\text {int }}$ & 0.0259 \\
\hline $\begin{array}{l}\text { Completeness to } \\
\theta=27.42^{\circ}(\%)\end{array}$ & 99.6 \\
\hline $\mathrm{GOF}$ on $F^{2}$ & 1.007 \\
\hline $\mathrm{R}_{1} / w \mathrm{R}_{2} \quad\left[\mathrm{~F}_{\mathrm{o}}^{2}>2 \sigma\left(\mathrm{F}_{\mathrm{o}}^{2}\right)\right]^{\mathrm{a}}$ & $0.0200 / 0.0460$ \\
\hline $\begin{array}{c}\mathrm{R}_{1} / w \mathrm{R}_{2} \\
\text { (all data) }\end{array}$ & $0.0204 / 0.0462$ \\
\hline Absolute structure parameter & $0.05(4)$ \\
\hline${ }^{\mathrm{a}} \mathrm{R}_{1}(F)=\sum \|\left|\mathrm{F}_{\mathrm{o}}\right|-\left|\mathrm{F}_{\mathrm{c}}\right|\left|/ \sum\right| \mathrm{F}_{\mathrm{c}}$ & $\left.\left.\mathrm{F}_{\mathrm{c}}^{2}\right)^{2} / \sum \mathrm{w}\left(\mathrm{F}_{\mathrm{o}}^{2}\right)^{2}\right]^{1 / 2}$ \\
\hline
\end{tabular}

Table S2. Selected bond lengths $(\AA)$ for $\mathrm{LiNbTeO}_{5}$.

\begin{tabular}{cccc}
\hline $\mathrm{Te}(1)-\mathrm{O}(1)$ & $1.896(8)$ & $\mathrm{Nb}(2)-\mathrm{O}(7) \# 6$ & $2.008(7)$ \\
$\mathrm{Te}(1)-\mathrm{O}(4)$ & $1.901(6)$ & $\mathrm{Nb}(2)-\mathrm{O}(8)$ & $1.753(7)$ \\
$\mathrm{Te}(1)-\mathrm{O}(9)$ & $1.891(8)$ & $\mathrm{Nb}(2)-\mathrm{O}(9)$ & $2.077(8)$ \\
$\mathrm{Te}(2)-\mathrm{O}(2)$ & $1.890(8)$ & $\mathrm{Nb}(2)-\mathrm{O}(10) \# 7$ & $2.212(9)$ \\
$\mathrm{Te}(2)-\mathrm{O}(5)$ & $1.875(8)$ & $\mathrm{O}(1)-\mathrm{Li}(1)$ & $2.00(2)$ \\
$\mathrm{Te}(2)-\mathrm{O}(10)$ & $1.885(7)$ & $\mathrm{O}(2)-\mathrm{Li}(1) \# 1$ & $1.90(2)$ \\
$\mathrm{Nb}(1)-\mathrm{O}(1) \# 2$ & $2.112(7)$ & $\mathrm{O}(3)-\mathrm{Li}(1) \# 1$ & $2.10(2)$ \\
$\mathrm{Nb}(1)-\mathrm{O}(2) \# 3$ & $2.222(9)$ & $\mathrm{O}(4)-\mathrm{Li}(2) \# 6$ & $2.13(2)$ \\
$\mathrm{Nb}(1)-\mathrm{O}(3)$ & $1.794(8)$ & $\mathrm{O}(5)-\mathrm{Li}(1) \# 4$ & $2.28(2)$ \\
$\mathrm{Nb}(1)-\mathrm{O}(5)$ & $2.011(9)$ & $\mathrm{O}(6)-\mathrm{Li}(1) \# 2$ & $2.04(2)$ \\
$\mathrm{Nb}(1)-\mathrm{O}(6)$ & $2.006(8)$ & $\mathrm{O}(7)-\mathrm{Li}(2) \# 8$ & $2.28(2)$ \\
$\mathrm{Nb}(1)-\mathrm{O}(7)$ & $1.906(7)$ & $\mathrm{O}(8)-\mathrm{Li}(2) \# 8$ & $1.97(2)$ \\
$\mathrm{Nb}(2)-\mathrm{O}(4) \# 5$ & $2.057(7)$ & $\mathrm{O}(9)-\mathrm{Li}(2) \# 1$ & $2.09(2)$ \\
$\mathrm{Nb}(2)-\mathrm{O}(6)$ & $1.962(8)$ & $\mathrm{O}(10)-\mathrm{Li}(2) \# 9$ & $1.98(2)$ \\
\hline
\end{tabular}

Symmetry transformations used to generate equivalent atoms:

$\begin{array}{lll}\# 1-\mathrm{x}, \mathrm{y}+1 / 2,-\mathrm{z}+1 & \# 2 \mathrm{x}+1, \mathrm{y}, \mathrm{z}+1 & \# 3-\mathrm{x}+2, \mathrm{y}-1 / 2,-\mathrm{z}+2 \\ \# 4 \mathrm{x}+2, \mathrm{y}, \mathrm{z}+1 & \# 5 \mathrm{x}+1, \mathrm{y}, \mathrm{z} & \# 6 \mathrm{x}-1, \mathrm{y}, \mathrm{z} \\ \# 7-\mathrm{x}+1, \mathrm{y}-1 / 2,-\mathrm{z}+2 & \# 8-\mathrm{x}+1, \mathrm{y}+1 / 2,-\mathrm{z}+1 & \# 9-\mathrm{x}+1, \mathrm{y}+1 / 2,-\mathrm{z}+2 \\ \# 10 \mathrm{x}-1, \mathrm{y}, \mathrm{z}-1 & \# 11-\mathrm{x}+2, \mathrm{y}+1 / 2,-\mathrm{z}+2 & \# 12-\mathrm{x}, \mathrm{y}-1 / 2,-\mathrm{z}+1 \\ \# 13 \mathrm{x}-2, \mathrm{y}, \mathrm{z}-1 & \# 14-\mathrm{x}+1, \mathrm{y}-1 / 2,-\mathrm{z}+1 & \end{array}$


Table S3. Atomic coordinates $\left(\times 10^{4}\right)$ and equivalent isotropic displacement parameters $\left(\AA^{2} \times 10^{3}\right)$ for $\mathrm{LiNbTeO}_{5} \mathrm{U}(\mathrm{eq})$ is defined as one third of the trace of the orthogonalized $\mathrm{U}_{\mathrm{ij}}$ tensor.

\begin{tabular}{ccccc}
\hline & $\mathrm{x}$ & $\mathrm{y}$ & $\mathrm{z}$ & $\mathrm{U}(\mathrm{eq})$ \\
\hline $\mathrm{Te}(1)$ & $-3074(1)$ & $2555(1)$ & $2943(1)$ & $7(1)$ \\
$\mathrm{Te}(2)$ & $12064(1)$ & $6248(1)$ & $11971(1)$ & $7(1)$ \\
$\mathrm{Nb}(1)$ & $7273(2)$ & $4526(1)$ & $9244(1)$ & $6(1)$ \\
$\mathrm{Nb}(2)$ & $2078(2)$ & $3698(1)$ & $5968(1)$ & $6(1)$ \\
$\mathrm{O}(1)$ & $-4528(15)$ & $3856(8)$ & $1471(9)$ & $7(2)$ \\
$\mathrm{O}(2)$ & $11019(14)$ & $7549(8)$ & $10375(8)$ & $10(2)$ \\
$\mathrm{O}(3)$ & $5879(15)$ & $6131(8)$ & $9296(9)$ & $15(2)$ \\
$\mathrm{O}(4)$ & $-6010(14)$ & $2457(8)$ & $4362(8)$ & $11(2)$ \\
$\mathrm{O}(5)$ & $10390(16)$ & $4860(7)$ & $10815(9)$ & $12(2)$ \\
$\mathrm{O}(6)$ & $4384(15)$ & $3546(8)$ & $8015(9)$ & $10(2)$ \\
$\mathrm{O}(7)$ & $9387(15)$ & $4660(7)$ & $7289(9)$ & $10(2)$ \\
$\mathrm{O}(8)$ & $3650(14)$ & $5045(8)$ & $5079(9)$ & $10(2)$ \\
$\mathrm{O}(9)$ & $-1068(16)$ & $3785(8)$ & $4225(9)$ & $13(2)$ \\
$\mathrm{O}(10)$ & $9424(15)$ & $6724(8)$ & $13476(9)$ & $14(2)$ \\
$\mathrm{Li}(1)$ & $-7470(40)$ & $2980(20)$ & $170(30)$ & $22(5)$ \\
$\mathrm{Li}(2)$ & $2660(40)$ & $570(20)$ & $5060(30)$ & $19(3)$ \\
\hline
\end{tabular}

Table S4. Anisotropic displacement parameters $\left(\AA^{2} \times 10^{3}\right)$ for $\mathrm{LiNbTeO}_{5}$. The anisotropic displacement factor exponent takes the form: $-2 \pi^{2}\left[\mathrm{~h}^{2} \mathrm{a}^{* 2} \mathrm{U} 11+\ldots+2 \mathrm{hka} * \mathrm{~b} * \mathrm{U} 12\right]$.

\begin{tabular}{ccccccc}
\hline $\mathrm{Te}(1)$ & $6(1)$ & $7(1)$ & $8(1)$ & $1(1)$ & $2(1)$ & $1(1)$ \\
\hline $\mathrm{Te}(2)$ & $7(1)$ & $6(1)$ & $9(1)$ & $0(1)$ & $1(1)$ & $0(1)$ \\
$\mathrm{Nb}(1)$ & $6(1)$ & $5(1)$ & $7(1)$ & $-1(1)$ & $2(1)$ & $1(1)$ \\
$\mathrm{Nb}(2)$ & $5(1)$ & $5(1)$ & $7(1)$ & $1(1)$ & $1(1)$ & $-1(1)$ \\
$\mathrm{O}(1)$ & $4(4)$ & $5(4)$ & $13(4)$ & $0(3)$ & $-2(3)$ & $2(3)$ \\
$\mathrm{O}(2)$ & $8(4)$ & $10(4)$ & $13(4)$ & $5(4)$ & $1(3)$ & $-1(4)$ \\
$\mathrm{O}(3)$ & $12(4)$ & $14(5)$ & $19(4)$ & $-4(4)$ & $1(3)$ & $4(4)$ \\
$\mathrm{O}(4)$ & $13(4)$ & $9(4)$ & $12(4)$ & $4(3)$ & $5(3)$ & $-1(4)$ \\
$\mathrm{O}(5)$ & $16(4)$ & $8(4)$ & $13(4)$ & $0(3)$ & $-1(3)$ & $-1(4)$ \\
$\mathrm{O}(6)$ & $15(4)$ & $8(4)$ & $8(3)$ & $-3(3)$ & $-1(3)$ & $1(3)$ \\
$\mathrm{O}(7)$ & $4(4)$ & $14(5)$ & $13(4)$ & $3(3)$ & $0(3)$ & $1(3)$ \\
$\mathrm{O}(8)$ & $10(3)$ & $9(3)$ & $11(2)$ & $0(2)$ & $3(2)$ & $-3(2)$ \\
$\mathrm{O}(9)$ & $9(4)$ & $14(4)$ & $17(4)$ & $-1(4)$ & $-1(3)$ & $-3(4)$ \\
$\mathrm{O}(10)$ & $10(4)$ & $15(5)$ & $17(4)$ & $0(3)$ & $7(3)$ & $4(4)$ \\
$\mathrm{Li}(1)$ & $16(11)$ & $27(14)$ & $24(11)$ & $12(9)$ & $3(8)$ & $-5(10)$ \\
$\mathrm{Li}(2)$ & $19(3)$ & $19(3)$ & $19(3)$ & $0(1)$ & $1(1)$ & $0(1)$ \\
$\mathrm{Te}(1)$ & $6(1)$ & $7(1)$ & $8(1)$ & $1(1)$ & $2(1)$ & $1(1)$ \\
\hline
\end{tabular}


Table S5. The direction and magnitude (Debye) of $\mathrm{LiO}_{5}, \mathrm{NbO}_{6}$ and $\mathrm{TeO}_{3}$ polyhedral dipole moments in $\mathrm{LiNbTeO}_{5}$.

\begin{tabular}{ccccc}
\hline Species & \multicolumn{4}{c}{ Direction and Magnitude (Debye) } \\
\cline { 2 - 5 } & $\mathrm{x}(\mathrm{a})$ & $\mathrm{y}(\mathrm{b})$ & $\mathrm{z}(\mathrm{c})$ & Total \\
$\mathrm{Nb}(1) \mathrm{O}_{6}$ & 1.77 & 4.20 & 2.70 & 5.30 \\
$\mathrm{Nb}(1) \mathrm{O}_{6}$ & -1.77 & 4.20 & -2.70 & 5.30 \\
$\mathrm{Nb}(2) \mathrm{O}_{6}$ & -1.38 & 0.32 & -2.35 & 2.74 \\
$\mathrm{Nb}(2) \mathrm{O}_{6}$ & 1.38 & 0.32 & 2.35 & 2.74 \\
$\sum \mathrm{Nb}-\mathrm{O}$ & 0 & 9.02 & 0 & 9.02 \\
$\mathrm{Te}(1) \mathrm{O}_{3}$ & -4.24 & -8.79 & 3.36 & 10.32 \\
$\mathrm{Te}(1) \mathrm{O}_{3}$ & 4.24 & -8.79 & -3.36 & 10.32 \\
$\mathrm{Te}(2) \mathrm{O}_{3}$ & 10.03 & -1.22 & 3.62 & 10.73 \\
$\mathrm{Te}(2) \mathrm{O}_{3}$ & -10.03 & -1.22 & -3.61 & 10.73 \\
$\sum \mathrm{Te}-\mathrm{O}$ & 0.00 & -20.02 & 0.00 & 20.02 \\
$\mathrm{Li}(1) \mathrm{O}_{5}$ & -1.34 & -3.93 & 0.68 & 4.21 \\
$\mathrm{Li}(1) \mathrm{O}_{5}$ & 1.34 & -3.93 & -0.68 & 4.21 \\
$\mathrm{Li}(2) \mathrm{O}_{5}$ & -1.09 & 0.60 & -2.62 & 2.91 \\
$\mathrm{Li}(2) \mathrm{O}_{5}$ & 1.09 & 0.60 & 2.62 & 2.91 \\
$\sum \mathrm{Li}-\mathrm{O}$ & 0 & -6.65 & 0 & 6.65 \\
\hline
\end{tabular}

Table S6. Measured refractive index and birefringence of $\mathrm{LiNbTeO}_{5}$ at different wavelengths.

\begin{tabular}{ccccc}
\hline Wavelength $(\mu \mathrm{m})$ & $n_{x}$ & $n_{y}$ & $n_{z}$ & $\Delta n=n_{z^{-}} n_{x}$ \\
\hline 0.6328 & 2.1018 & 2.1208 & 2.1849 & 0.0831 \\
1.539 & 2.0491 & 2.0576 & 2.1020 & 0.0529 \\
\hline
\end{tabular}




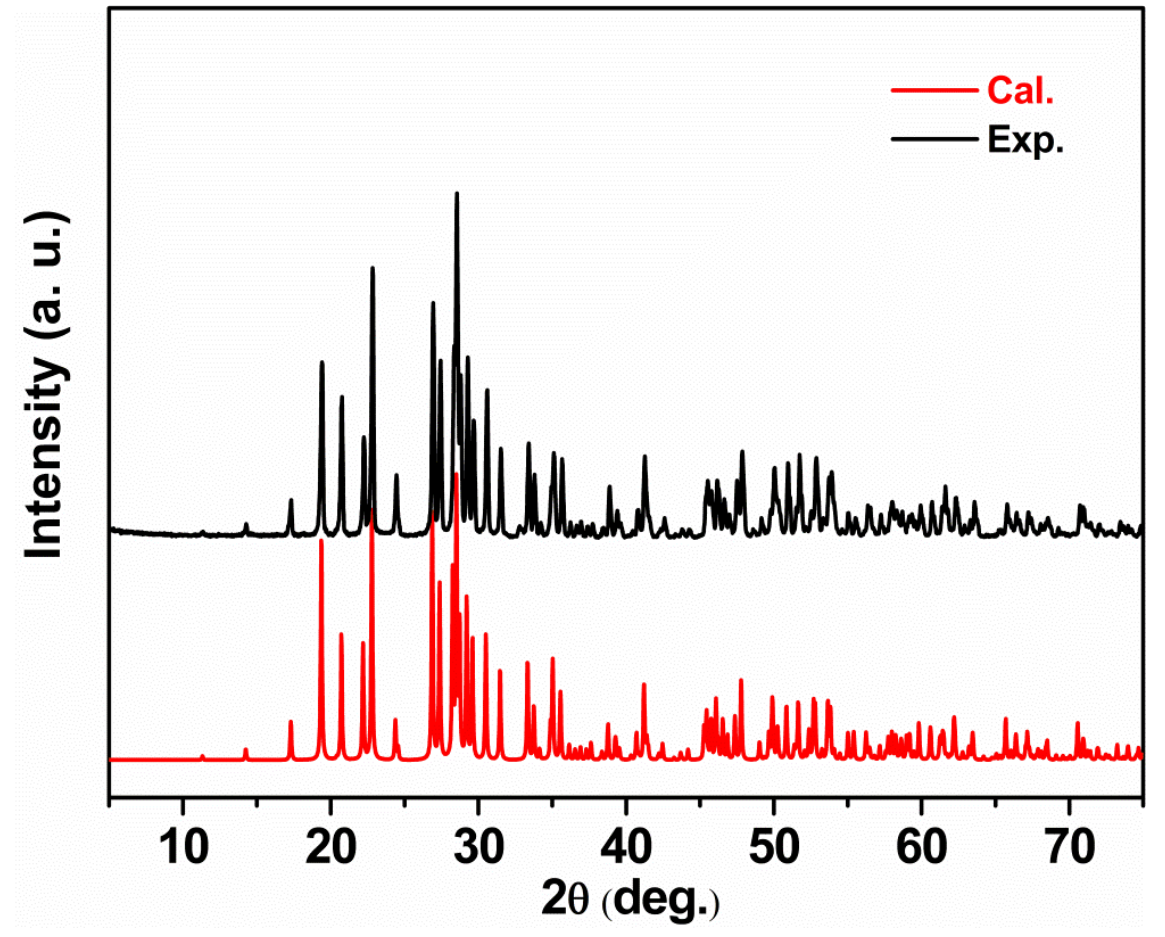

Figure S1. Calculated and experimental powder X-ray diffraction patterns of $\mathrm{LiNbTeO}_{5}$.

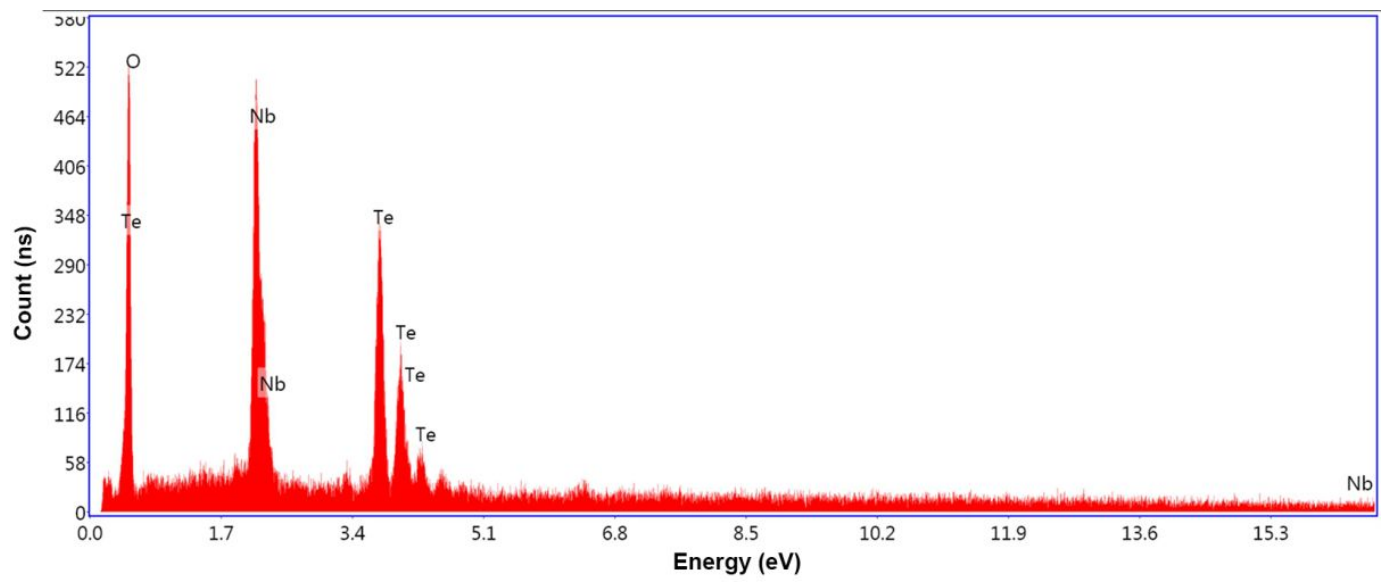

Figure S2. EDS analysis for $\mathrm{LiNbTeO}_{5}$. 


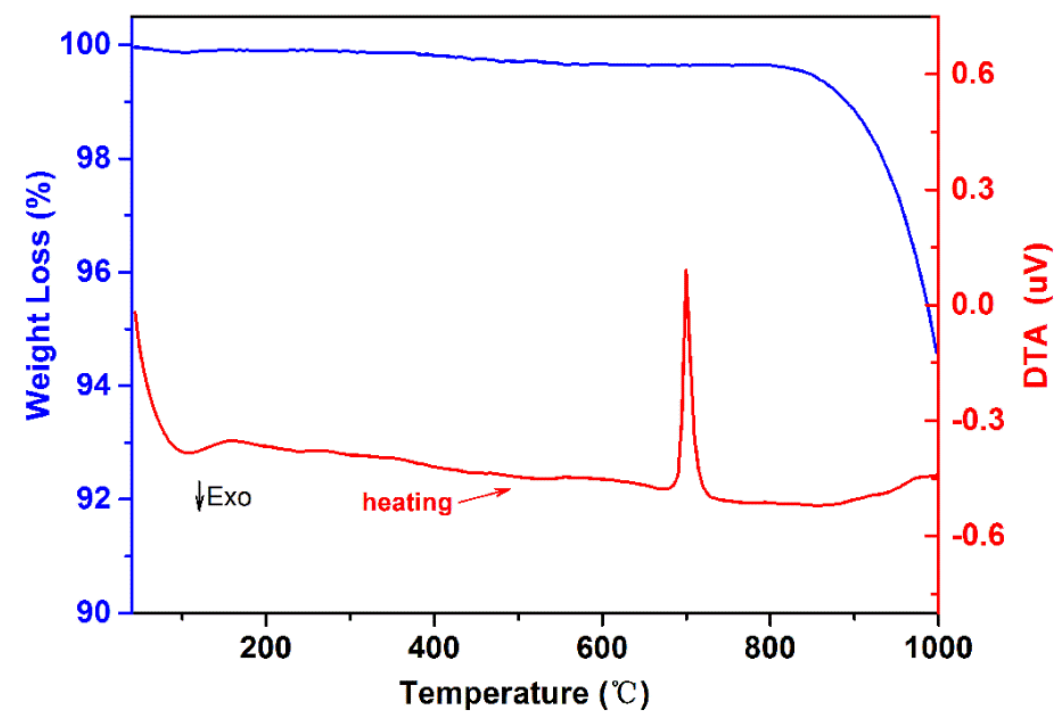

Figure S3. Thermal analysis curves of $\mathrm{LiNbTeO}_{5}$.

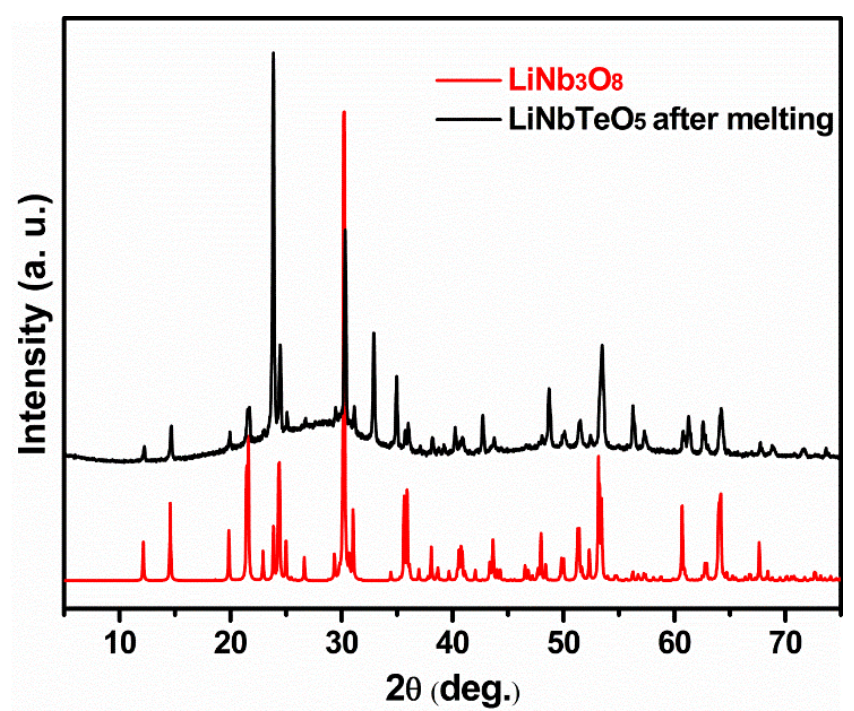

Figure S4. The powder XRD patterns of the residue after melting for $\mathrm{LiNbTeO}_{5}$.

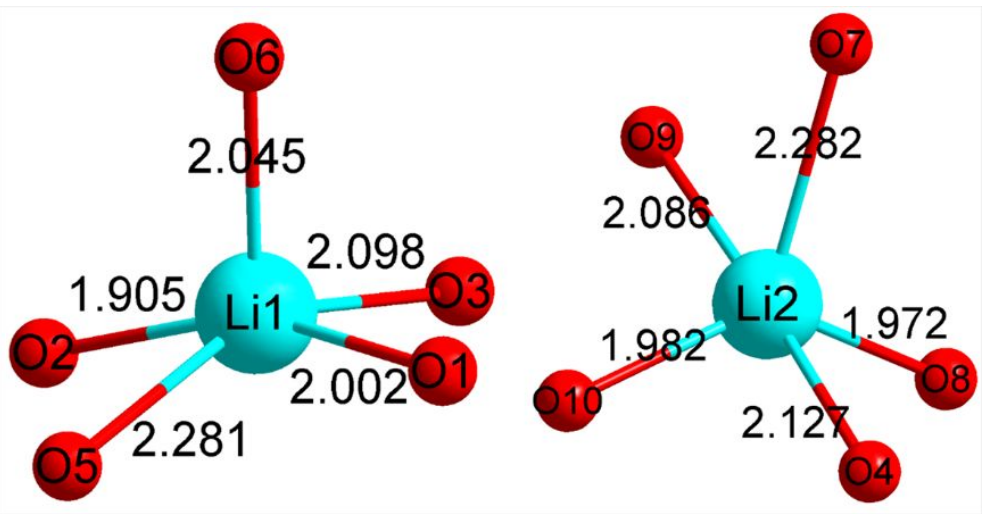

Figure S5. The $\mathrm{LiO}_{5}$ polyhedra in $\mathrm{LiNbTeO}_{5}$ with bond length $(\AA)$ marked out. 

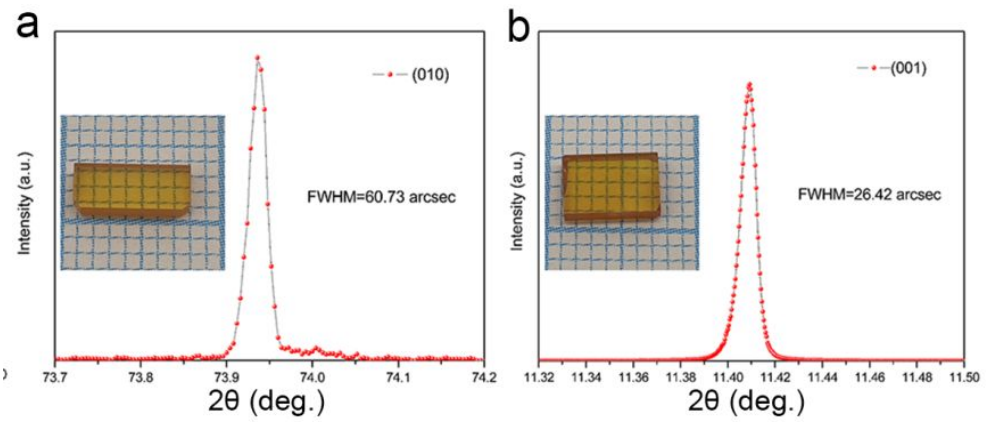

Figure S6. High-resolution XRD rocking curve of a (010)- and (001)-oriented $\mathrm{LiNbTeO}_{5}$ wafer (inset) with FWHM value marked out

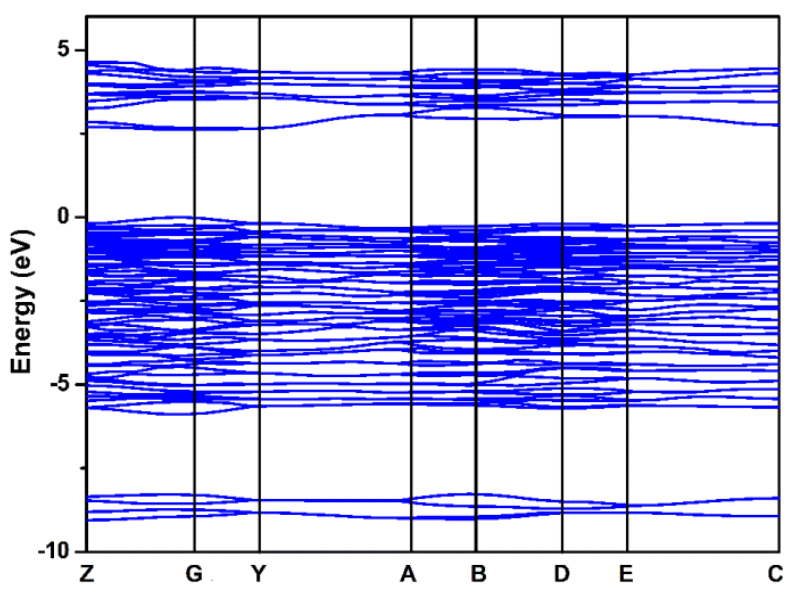

Figure S7. Electronic band structure of $\mathrm{LiNbTeO}_{5}$.

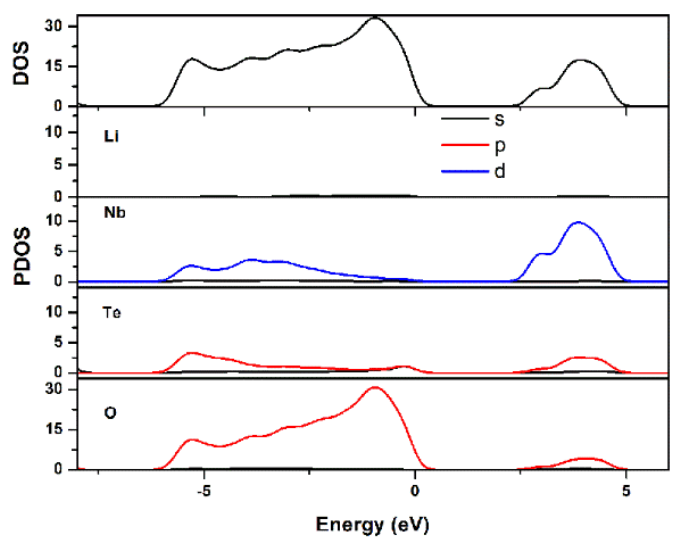

Figure S8. The partial and total densities states of $\mathrm{LiNbTeO}_{5}$. 